\title{
Pre-1938 Product
}

National Cancer Institute

\section{Source}

National Cancer Institute. Pre-1938 Product. NCI Thesaurus. Code C99176.

A compound or product that was available prior to the passage of the 1938 Federal Food, Drug and Cosmetic Act. 